Chimia 45 (1991) 304

(c) Schweiz. Chemiker-Verband; ISSN 0009-4293

\section{Experiments in Color}

\author{
Herbert W. Roesky*
}

Chemistry can be defined as the integrated study of the preparation, properties, and reactions of the chemical elements and their compounds and of the systems they form. Therefore, it is quite obvious that chemical demonstrations lead to fundamental knowledge in chemistry. Furthermore people are mainly attracted to chemistry by exciting experiments. However, more than $99 \%$ of all speakers in chemistry are not using chemical demonstrations. What are the reasons? From my experience with chemica

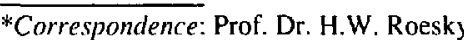

Tammannstrasse 4

D-3400 Göttingen demonstrations, it takes a long time to optimise an experiment and to be well prepared to ensure success. In the case one experiment fails to react in the proper way, people are enjoying and talking about this one more than about the rest of the well performed ones. In addition, the chemical community has not created awards to encourage scientists to do chemical demonstrations in the public.

In my opinion, the most important purpose of chemical demonstrations is to communicate with the audience and to appreciate the intellectual excitement and challenge of chemistry. I hope that the following 24 demonstrations will contribute to the bright colors of this symposium.
1. Oscillating colors

2. Ice cream and cherry juice

3. Dancing fireball

4. Barking dog

5. Disco colors

6. Bang box

7. Fractal structures

8. Artificial beer

9. Artificial softdrink

10. Chemiluminescence

11. A colored water test of Montreux water

12. Singlet oxygen

13. Start of a rocket

14. Two-color chemiluminescence

15. Landolt reaction

16. Red and blue ink

17. Three-color oscillating reaction

18. Love story

19. Belousov reaction

20. Chemical garden

21. Blue bottle

22. Colored structures in solution, self-organization

23. Silver mirror

24. Recycling
Chimia 45 (1991) 304-307

(C) Schweiz. Chemiker-Verband: ISSN 0009-4293

\title{
The Functional Dyes - Definition, Design, and Development
}

\author{
John Griffiths*
}

\section{What Are Functional Dyes?}

For more than a century, new dye research has been driven by the needs of the textile industry, but the 1970's saw a major change in this situation. For economic and (increasingly) ecological reasons a period of rationalisation of the dye making industries took place, which continues today, and the search for new textile dyes is now seen generally to be of low priority. However, from about the same period new technologies arose, stimulated by rapid advances in materials, microelectronics and lasers, and many of these were dependent on highly

*Correspondence: Dr. J. Griffiths Department of Colour Chemistry University of Leeds

Leeds LS2 9JT, U.K. specialised dyes. The function of these dyes was more than merely aesthetic, and thus the term functional dye was coined to distinguish them from conventional colorants. They have presented the dye chemist with many new challenges, and research into these high technology materials continues apace. This intense interest is demonstrated by the success of the first ever international conference on functional dyes which was held in Osaka in 1989 and which attracted around 600 participants. A second is planned for 1992.

It could be argued that any commercial colorant is functional, otherwise it would necessarily be of no commercial interest. However, we can exclude dyes from our definition that are used solely for aesthetic coloration purposes, thus making the term functional dye more precise. Other designations encountered in connection with functional dyes are speciality dyes and hi-tech dyes, but these are much less specific terms.
Although such terms embrace all functional dyes, not all speciality and high-technology dyes can be classed as functional dyes. $E . g$., smoke dyes are clearly 'specialised', and thermal transfer dyes used in electronic photography are 'hi-tech'. However, neither are strictly speaking functional dyes as in both instances the dyes are used solely to impart colour. Many dyes show dual functionality, and e.g. fluorescent dyes as used in textiles and inks are simply 'specialised' colorants, but when used as laser dyes are then true 'functional' dyes.

It is also important to consider what we mean exactly by the word dye when we talk of these functional colorants, as the traditional concept of a dye can become severely stretched. E.g. functional IR dyes with practically no colour are known, and even colourless UV absorbing compounds used as light attentuators in high resolution microphotolithographic resist resins are popularly referred to as 'dyes'. For convenience and generality, therefore, a functional dye may be thought of as an organic molecule whose electronic structure is such that electromagnetic radiation can be absorbed readily by electronic excitation, and whose function is directly or indirectly dependent on the potential of the molecule for such excitation. It follows that the vast majority of functional dyes will contain conjugated $\pi$-electron systems.

We can classify functional dyes according to the molecular opto-electronic process that their function is dependent on. These possible mechanisms are summarised in the 